\title{
Precipitating Factors for the Development of Diabetic Ketoacidosis in a Tertiary Care Hospital in Bahrain
}

\author{
Mandeel, Fatema H. ${ }^{\text {; }}$ Radhi, Husain T. ${ }^{\text {; }}$, Sarwani, Aysha A. ${ }^{\text {; }}$ Alsadah, Ahmed H. ${ }^{2}$ \\ ${ }^{1,}$ Department of Endocrinology, Salmaniya Medical Complex, Bahrain, \\ ${ }^{2}$ Department of Accident and Emergency, Qatif, KSA \\ *Corresponding author: Fatema Hussain Mandeel, Mobile: +973 33931910, email: dr.mandeel124@gmail.com
}

\begin{abstract}
Background: Diabetic ketoacidosis is a commonly presenting complication of diabetes occurring commonly in type 1 and occasionally in type 2 DM patients as well, the precipitating factors for which are varied and only a few studies have explored this.

Objective : To review the causative factors that lead to the occurrence of DKA among the adult age group of patients in Bahrain.

Methods: The study was conducted in a retrospective, descriptive method in Salmaniya Medical Complex, Bahrain. The age group included consisted of adult patients of age 14 years and onwards, during a period of two years 20172018. Patient demographic data as well as related scientific data during the hospital stay were collected.

Results: A major precipitating factor was non-compliance (62 patients) with treatment regimen followed by infectious causes (26 patients). Poor diabetic control also caused a high occurrence of DKA (16 patients), as well as disruption in insulin supply, lead to DKA in patients on an insulin pump (3 patients). A high readmission rate was noted in the admission analysis due to repetitively occurring causative factors.

Conclusion:Preventing the occurrence of precipitating factors of DKA, through patient education and support can decrease the occurrence of this preventable complication of diabetes, leading to decrease morbidity and mortality related to DKA.
\end{abstract}

Keywords: Diabetes, Diabetic ketoacidosis, Precipitating factors.

Abbreviation,DM: Diabetes Mellitus;DKA: Diabetic ketoacidosis.

\section{INTRODUCTION}

Diabetic ketoacidosis (DKA) is a life-threatening complication of diabetes mellitus (DM). It is commonly associated with type 1 diabetes, but it is not uncommon in some patients with type 2 diabetes. It resulted from absolute or relative insulin deficiency and it is characterized by hyperglycemia, ketonemia, and acidosis ${ }^{1}$.

DKA can be classified into mild, moderate, or severe based on the metabolic acidosis severity and mental status alteration. The most common precipitant for ketoacidosis was omission/error of insulin administration followed by acute illness ${ }^{2}$.

Although mortality rates had fallen significantly over the past 20 years from $7.96 \%$ to $0.67 \%$, the mortality rate is still high in non-hospitalized patients and developing countries ${ }^{3}$. DKA is a serious but largely preventable acute complication of diabetes mellitus. Identifying precipitating factors and increase awareness will prevent admission and associated mortality and morbidity ${ }^{4}$.

\section{MATERIAL AND METHODS}

This was a retrospective and descriptive study conducted at a tertiary academic center, Salmaniya Medical Complex, Bahrain. The study was approved by the secondary health care research committee at the study site. Inclusion criteria for study involvement were admission to the medical center between 2017 and 2018 with the diagnosis of DKA. Patients younger than 14 years old not fulfilling the criteria of DKA were ruled out during admission.

\section{Statistical analysis:}

The data collection form included the patient's age, gender, nationality, type of diabetes, comorbidities, HbA1c, readmissions, and precipitating factors. IBM SPSS 25 and $\mathrm{R}$ studio 1.1.14 were used to analyze the collected data. The variable relations were investigated with the proper statistical methods (Chi-square test and A NOVA).

\section{Ethical approval:}

The study was approved by Secondary Health Care Research Sub Committee in Salmaniya Medical Complex and an informed written consent was taken from each participant in the study.

\section{RESULTS \\ Patient characteristics}

A total of 224 patients were enrolled in the current study, $58.5 \%$ of whom were males while $41.5 \%$ were females. The age group ranged from 14 to more than 76 years old.

The majority of the patients were Bahraini (80.8\%) while the rest were from 13 other nationalities $(19.1 \%)$ (Table 1). Nearly two-thirds of them have no comorbidities while a third has associated comorbidities $(37.1 \%)$. 
Table 1. Demographic characteristics of DKA patients

\begin{tabular}{|c|c|c|c|}
\hline Variable & Categories & $\mathbf{N}$ & $\%$ \\
\hline \multicolumn{4}{|c|}{ Gender } \\
\hline & Male & 131 & $58.5 \%$ \\
\hline & Female & 93 & $41.5 \%$ \\
\hline \multicolumn{4}{|c|}{ Nationality } \\
\hline & BAH & 181 & $80.8 \%$ \\
\hline & Non-BAH & 43 & 19.2 \\
\hline \multicolumn{4}{|c|}{ Age group } \\
\hline & $14-30$ & 118 & $52.7 \%$ \\
\hline & $31-45$ & 71 & $31.7 \%$ \\
\hline & $46-60$ & 25 & $11.2 \%$ \\
\hline & $61-75$ & 9 & $4.0 \%$ \\
\hline & $>76$ & 1 & $0.4 \%$ \\
\hline
\end{tabular}

\section{Demographic characteristics of DKA patients}

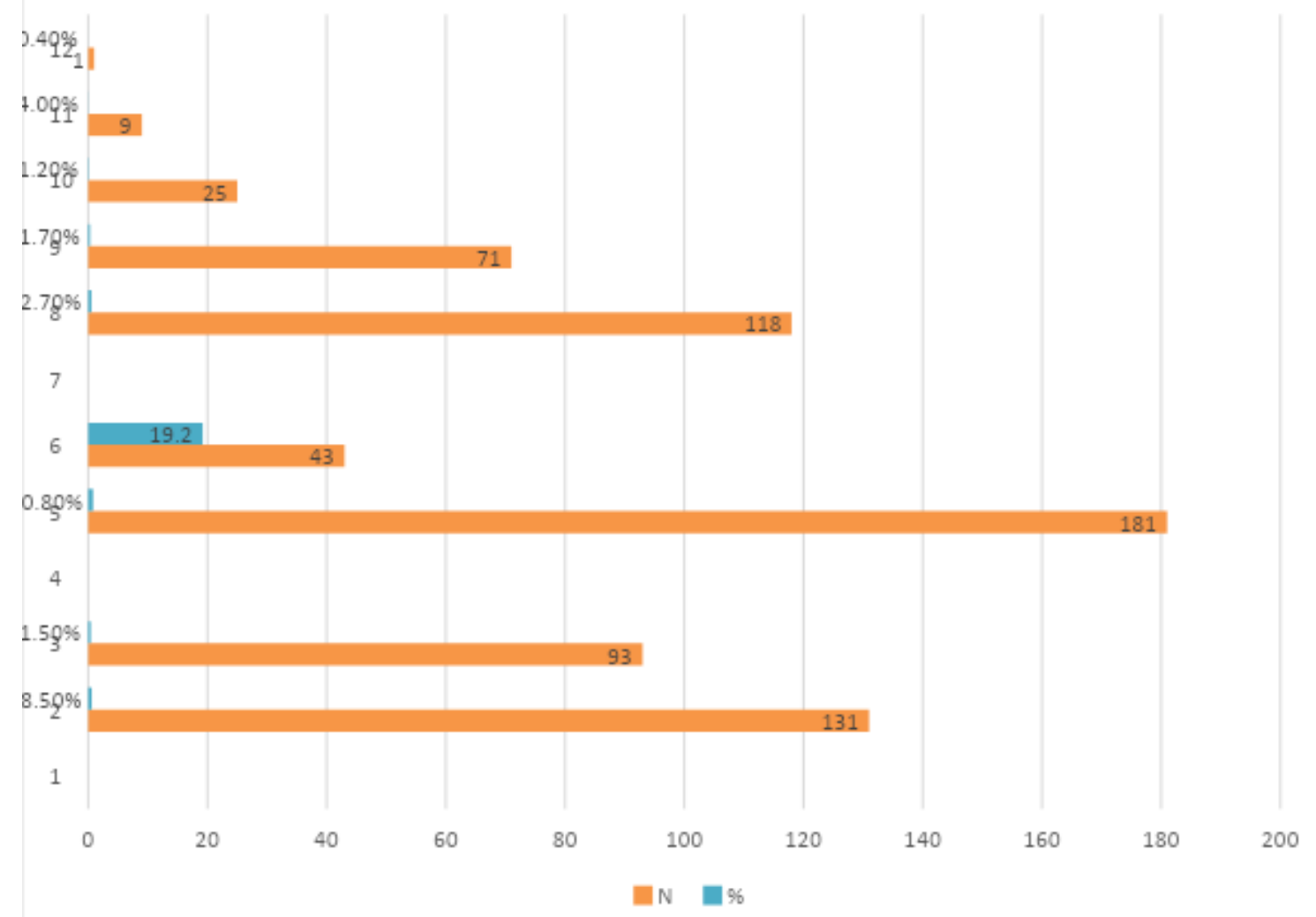

\section{Diabetes characteristics}

We categorize the patients per diabetes mellitus type. Newly diagnosed DM contributing for $14.3 \%$, type 1 DM for $65.6 \%$ and type $2 \mathrm{DM}$ for $20.1 \%$ (Table 2).

Table 2. Type of diabetes and comorbidities 


\section{Type of diabetes and comorbidities}

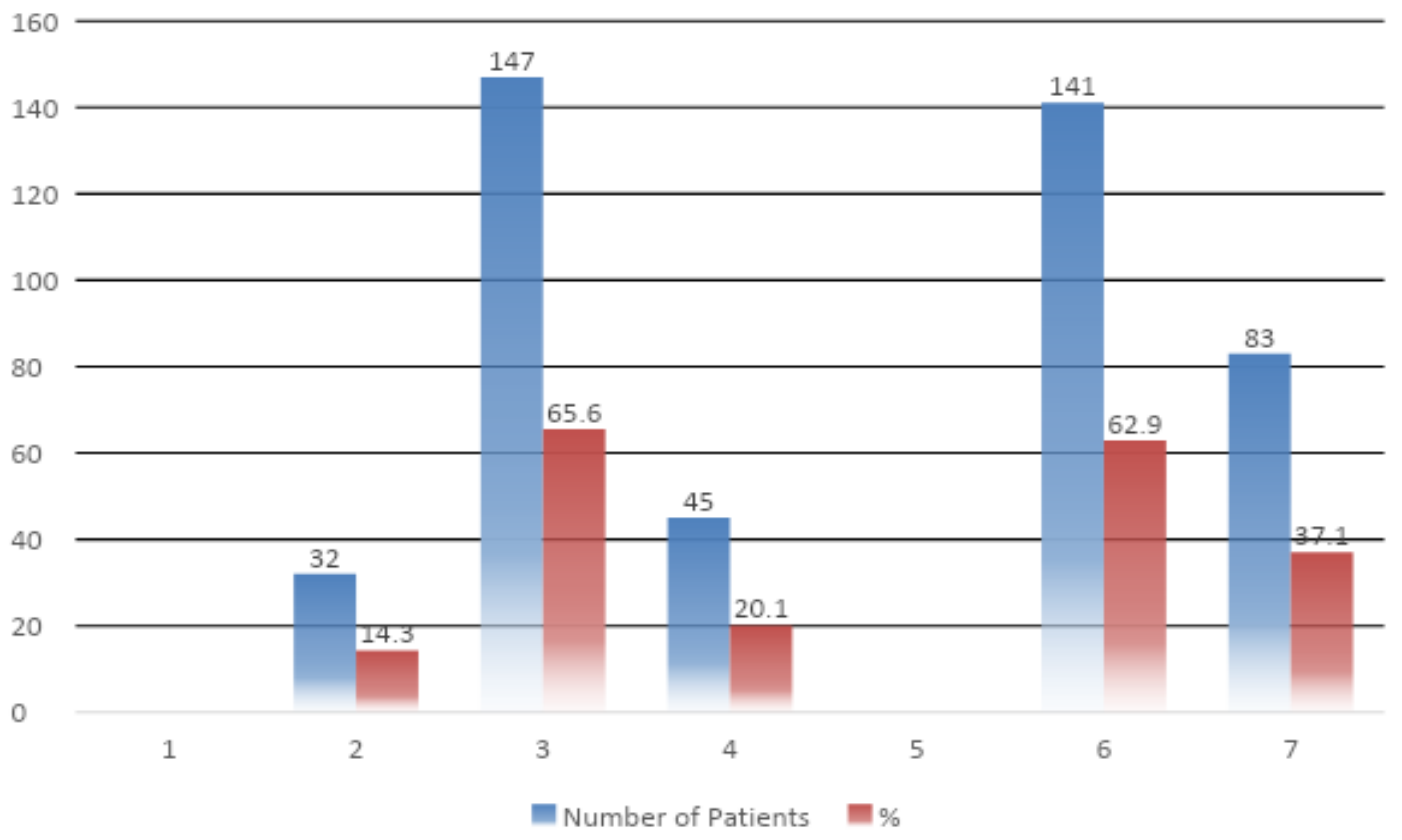

At presentation gastrointestinal symptoms were most common in DKA patients. Vomiting is the main symptom followed by abdominal pain and nausea (see table 3 for details).

Table 3. Presenting symptoms of DKA patients

\begin{tabular}{|c|c|c|c|}
\hline Type of DM & Abdominal pain & Nausea & Vomiting \\
\hline New & $10(31.3 \%)$ & $10(31.3 \%)$ & $14(43.8 \%)$ \\
\hline Type 1 & $67(46.6 \%)$ & $29(19.7 \%)$ & $89(60.5 \%)$ \\
\hline Type 2 & $17(37.8 \%)$ & $11(24.4 \%)$ & $19(42.2 \%)$ \\
\hline p-value & $\mathbf{0 . 2 7 0}$ & $\mathbf{0 . 3 4 0}$ & $\mathbf{0 . 0 4 1}$ \\
\hline
\end{tabular}

*Statistically significant difference with Chi-square test $(X 2=6.4, d f=2, p=0.041)$ at Alpha 0.05

The mean RBS and HbA1c on admission were documented. The newly diagnosed group had the lowest HbA1c on average (91.4) and type $2 \mathrm{DM}$ had the highest level (102.2) (Table 4).

Table 4. Glycemic control profile on admission

\begin{tabular}{|c|c|c|c|c|}
\hline & New & Type 1 & Type 2 & P-value \\
\hline RBS Mean $( \pm$ SD) & $26.7( \pm 12.3)$ & $27.7( \pm 9.6)$ & $28.7( \pm 10.1)$ & 0.629 \\
\hline HbA1c Mean $( \pm$ SD) & $91.4( \pm 35.1)$ & $92.9( \pm 25.7)$ & $102.2( \pm 37.9)$ & 0.300 \\
\hline
\end{tabular}

Around $93.5 \%$ of the readmissions occurred for type $1 \mathrm{DM}$ patients, $6.5 \%$ for type $2 \mathrm{DM}$, and $0 \%$ for the newly diagnosed DM (Table 5).

Table 5. Readmission among DKA patients

\begin{tabular}{|c|c|c|}
\hline DM type & $\mathrm{n}$ & $\%$ \\
\hline New & 0 & $0 \%$ \\
\hline Type 1 & 29 & $93.5 \%$ \\
\hline Type 2 & 2 & $6.5 \%$ \\
\hline p-value & \multicolumn{2}{|c|}{} \\
\hline
\end{tabular}

The statistically significant difference with the Chi-square test of independence $\left(X^{2}=6.3, d f=1, p=0.011\right)$ 


\section{Readmission among DKA patients}

35
30
25
20
15
10
5
0

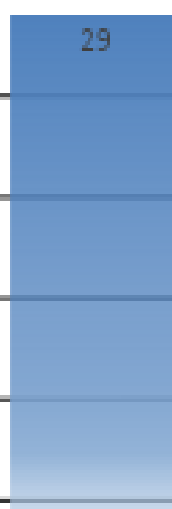

Type 1

In addition to their principle diagnosis as DKA, the predominant precipitating factor was non-compliance with medications, as it was reported in (62) patients, followed by infection (26), high HGT despite taking medication (16), and continuous subcutaneous insulin pump mechanical problem (3), Other precipitating factors are demonstrated in ( Table 6).

Table 6. Precipitating factors of DKA

\begin{tabular}{|c|c|c|c|c|c|c|}
\hline & \multicolumn{2}{|c|}{ New } & \multicolumn{2}{c|}{ Type 1 } & \multicolumn{2}{c|}{ Type 2 } \\
\hline Infection & 26 & $68.4 \%$ & 12 & $13.7 \%$ & 9 & $28.1 \%$ \\
\hline Non-compliance & 0 & & 62 & $71.2 \%$ & 15 & $46.9 \%$ \\
\hline High HGT & 10 & $26.3 \%$ & 1 & $1.1 \%$ & 4 & $12.5 \%$ \\
\hline Missed food & 0 & & 5 & $5.7 \%$ & 2 & $6.3 \%$ \\
\hline Pancreatitis & 2 & $5.2 \%$ & 0 & & 0 & \\
\hline Alcohol & 0 & & 0 & & 1 & $3.1 \%$ \\
\hline Insulin pump malfunction & 0 & & 3 & $3.3 \%$ & 0 & \\
\hline Cardiac ischemia & 0 & & 0 & & 1 & $3.1 \%$ \\
\hline Seizure & 0 & & 3 & $3.4 \%$ & 0 & \\
\hline Steroid use & 0 & & 1 & $1.1 \%$ & 0 & \\
\hline Total & $\mathbf{3 8}$ & & $\mathbf{8 7}$ & & $\mathbf{3 2}$ & \\
\hline
\end{tabular}




\section{DISCUSSION}

Our study revealed that there was no major difference seen in the age, gender, and nationality distribution presenting with DKA with both males and females comprising a significant number, males contributing to $58.5 \%$ whilst the females being $41.55 \%$. Bahrainis were more compared to nonBahrainis ( $\mathrm{n}=181,30.8 \%$ vs non-Bahrainis $\mathrm{n}=43$, $19.1 \%$ ) but no statistically significant difference was seen (Table 1).

Younger patients in the age group of 14- 30 years made up most the subjects' mean age being 31.2 years, followed by the age group 31-45 years, with a decreasing trend in the increasing age groups.

In a study by Ahuja, the mean age of DKA presentation was similar to what we found in our study i.e. young adults mean age being $19 \pm 7$ years (age group: 7-24 years)., however, they included the pediatric age group which was excluded in our study. The gender distribution was comparable to our study with women comprising $41.9 \%$, and men comprising $58.1 \%^{5}$.

147 patients (65.6\%) admitted were those with type $1 \mathrm{DM}$ while 45 (20.1) were those having type $2 \mathrm{DM}$, and $32(14.3 \%)$ were newly diagnosed coming with the first presentation of DKA (Table 2). Co-morbidities i.e. hypertension, dyslipidemia, coronary artery disease, chronic kidney disease among others were present in 83 (37.1) patients compared to 141 patients (62.9) without any comorbidities.

Y.XU study in China involved 643 patients with DKA. 308 patients (47.9\%) with type 1 DM, 249 with $(45.7 \%)$ with type $2 \mathrm{DM}$, and 41 patients with $(6.4 \%)$ with atypical diabetes ${ }^{6}$. DM type 1 patients are at risk of developing DKA due to absolute insulinopenia which leads to increase gluconeogenesis, lipolysis, ketogenesis, and decrease glycolysis ${ }^{7}$.

Comparing to other articles, the presentation was variable among the patients, with gastrointestinal symptoms being the most common, vomiting being the main complaint (146.5\%), followed by abdominal pain $(115.7 \%)$, and then nausea $(75.4 \%)$ (Table 3$)^{8}$.

The mean RBS on admission in all the patient groups was comparable (Table 4). In type 1 DM patients was 27.2 , in type 2 was 28.7 , and in the newly diagnosed was $27.4 \mathrm{mmol}$. The average $\mathrm{HbA} 1 \mathrm{c}$ on presentation was also comparable showing poor control with no statistically significant difference. Comparing to Holmes-Walker D, the effect of a transition support program in young DM patients revealed the initial mean $\mathrm{HbA} 1 \mathrm{c}$ was $9.3 \%$ +$2.17 \%^{9}$.

Readmissions were seen to be very common in patients presenting with DKA (Table 5). The causative factors kept on recurring in the majority of the type $1 \mathrm{DM}$ patients causing for high readmission rate. It was seen that $93.5 \%$ readmission rate overall was from type $1 \mathrm{DM}$ patients. For example, younger age at DKA onset has been associated with a high risk of DKA recurrence suggests that this group of patients are more vulnerable to develop hyperglycemic crisis $^{1^{0}}$.

Most studies demonstrated that the most common precipitating factors that came to the forefront were inadequate insulin therapy whether omitted or insufficient insulin regimen or presence of infection ${ }^{8},{ }^{11}$, followed by high HGT despite taking the doses and the other causes included alcohol intake, pancreatitis, pump malfunction and others (Table 6). Mechanical problems with continuous subcutaneous insulin infusion devices (i.e. blocked pump, kinked cannula) can precipitate DKA, however, with technology improvement and patient education, the incidence of DKA has been declining in insulin pump patients $^{12}$.

Other precipitating factors include cerebrovascular and renal accidents, myocardial ischemia, pancreatitis, depression, alcohol, and illicit drug use. Also, numerous underlying medical illnesses and medications start the release of counterregulatory hormones such as corticosteroids, sympathomimetics agents, thiazide diuretics, anticancer medications, and antipsychotic drugs, which may precipitate DKA ${ }^{1}$. Most recently, sodium-glucose co-transporter 2 (SGLT-2) inhibitors e.g. canagliflozin, dapagliflozin, and empagliflozin have emerged as triggers for $\mathrm{DKA}^{13}$.

\section{ACKNOWLEDGMENT}

The authors thank Mr. Ridha Al-Hammam for his data management support and st Limitation of study.

The study is limited to only one of the main hospitals in Bahrain and the limited study period of two years.

\section{CONCLUSION}

In conclusion, DKA is a serious but largely preventable complication. Several precipitating factors have been identified. Medication noncompliance and infection are the most common risk factors to develop DKA.

Those at-risk individuals during the first DKA admission need target intervention such as intensified pre-discharge diabetic education. Instruction of insulin use/ medication adherence, social support, the involvement of family members in medical treatment, education about sick day management, close follow up is very important as detection of symptoms. It has been shown that three-monthly visits to the endocrine clinic will reduce the number of ER admissions for DKA . Launching of awareness and education programs in the public and through social media platforms with the aim of improving DM patient's quality of life, preventing further admission and associated morbidity and mortality. 


\section{DISCLOSURE}

There is no grant support of other assistance to declare for this article, and the authors have no conflicts of interest to declare.

\section{REFERENCES}

1. Kitabchi A, Umpierrez G, Murphy M, Kreisberg R (2006): Hyperglycemic Crises in Adult Patients with Diabetes: A consensus statement from the American Diabetes Association. Diabetes Care, 29(12): 2739-2748.

2. Isidro M , Jorge S (2010): Recreational drug abuse in patients hospitalized for diabetic ketosis or diabetic ketoacidosis. Acta Diabetologica, 50(2): 183-187.

3. Kohler K, Levy N (2014): Management of Diabetic Ketoacidosis: A Summary of the 2013 Joint British Diabetes Societies Guidelines. Journal of the Intensive Care Society, 15(3):222-225.

4. Bradford A , Crider C, Xu X, Naqvi S (2017): Predictors of Recurrent Hospital Admission for Patients Presenting with Diabetic Ketoacidosis and Hyperglycemic Hyperosmolar State. Journal of Clinical Medicine Research, 9(1): 35-39.

5. Ahuja W , Kumar N , Kumar S, Rizwan A (2019): Precipitating Risk Factors, Clinical Presentation, and Outcome of Diabetic Ketoacidosis in Patients with Type 1 Diabetes. Cureus, 11(5): e4789.
6. Xu Y, Bai J, Wang G et al.( 2015): Clinical profile of diabetic ketoacidosis in tertiary hospitals in China: a multicenter, clinic-based study. Diabetic Medicine, 33(2): 261-268.

7. Kitabchi A, Nyenwe E (2006): Hyperglycemic Crises in Diabetes Mellitus: Diabetic Ketoacidosis and Hyperglycemic Hyperosmolar State. Endocrinology and Metabolism Clinics of North America, 35(4): 725-751.

8. Singh H , Saroch A , Pannu A et al. (2019): Clinical and biochemical profile, precipitants and prognostic factors of diabetic ketoacidosis: A retrospective study from a tertiary care center of north India. Diabetes \& Metabolic Syndrome: Clinical Research \& Reviews, 13(4): 2357-2360.

9. Holmes-Walker D, Llewellyn A, Farrell K (2007): A transition care program that improves diabetes control and reduces hospital admission rates in young adults with Type 1 diabetes aged 15- 25 years. Diabetic Medicine, 24(7): 764-769.

10. Kim H, Ross JR, Boockvar K (2010): Schedule and Unscheduled Hospital Readmissions among Diabetes Patients. Am J Manag Care, 16(10): 760-767.

11. Cooper H, Tekiteki A, Khanolkar M, Braatvedt G (2015): Risk factors for recurrent admissions with diabetic ketoacidosis: a case-control observational study. Diabetic Medicine, 33(4): 523-528.

12. Weissberg-Benchell J, Antisdel-Lomaglio J, Seshadri R (2003): Insulin Pump Therapy: A meta-analysis. Diabetes Care, 26(4): 1079-1087. 